\title{
Interactive comment on "The incorporation of the
} Tripleclouds concept into the $\delta$-Eddington \section{two-stream radiation scheme: solver} characterization and its application to shallow cumulus clouds" by Nina Črnivec and Bernhard Mayer

\section{Lazaros Oreopoulos (Referee)}

lazaros.oraiopoulos@nasa.gov

Received and published: 11 June 2020

This paper introduces a new formulation for the triple cloud (TC) scheme which provides a simple framework for dealing with subgrid cloud heterogeneity in GCM radiative transfer calculations. The new formulation has to do with how the thick portion of the cloud in triple clouds (triple = thick cloud, thin cloud, clear) is treated in terms of overlap for adjacent cloud layers. Fig. 9 also nicely summarizes differences in the way 
upward reflected fluxes are distributed to the cloud layer above. The authors claim that a new element (even call it "pioneering") of their work is validation using surface fluxes and heating rates, as opposed to previous TOA only flux validation, but this is a trivial consideration, since the previous triple scheme can do that too, it's just never been presented. The basic structure of the paper is that the modified TC scheme is introduced and then applied on a simplified (according to TC rules) version of an LES cumulus field, both for SW (different solar angles) and then LW. The results are compared against the ICA calculation (should be better) and a full Monte Carlo calculation that accounts for full 3D cloud structure (should be even better); also against a standard non-TC GCM scheme (which should be worse). Various choices on how to distribute the cloud layer liquid water content to the thick and thin regions are presented. Results are slightly different between the different TC versions, but almost always better than a traditional non-TC GCM RT scheme, except perhaps for surface LW fluxes (which are though very small in a net flux sense because the upward and downward LW fluxes for a cloud field with low bases are very similar).

Overall, this is a good paper that I enjoyed reading and needs only some minor fixes (per comments below) to see the light of day as a proper ACP paper.

Here are some specific comments:

The assumption that the thicker part of the cloud will be towards the center is reasonable, but demonstrating that with fig. 3 is almost irrelevant because that figure shows the centers of multiple small clouds, which the GCM does not represent. The GCM implicitly only has a single cloud in a 50-100 km grid cell.

It is not clear how clouds overlap is treated when the cloud layers are separated by a clear layer. Is it random overlap then? How would one of the Fig. 8 panels look if there was a single clear layer between the two cloudy layers? So, is the overlap only considered for neighboring cloudy layer pairs? Are pairs of cloudy layers that are distant completely independent even if there is no clear layer in-between? In other words, only 
a pair-wise coupling of fluxes is considered? Generalized (exponential-random) overlap can overlap any pair of clouds, but of course explicit radiative treatment is messy (if not impossible) without subcolumns.

I'm not convinced that this method is better than McICA because the latter can operate on any subcolumns which can be generated with more realistic rules for overlap and LWC subgrid variability (and its overlap). I mean, if exponential-random agrees better with observations, why not try to use it? The authors state that the McICA noise may be undesirable and impactful, and that's perhaps true, but perhaps this is less important than achieving smaller systematic biases? I don't buy the argument in lines 72-74 that fewer spectral intervals will make McICA worse. This seems to assume that you have to produce only as many columns as g-points so that each column is paired with one g-point, but this doesn't have to be the case. One can easily generate Nc-multiple of g-point subcolumns (i.e., a total $\mathrm{Nc}^{*} \mathrm{Ng}$ subcolumns) of so that the same spectral point operates on Nc subcolumns. This will reduce the noise. The fewer g-points, the less the McICA noise, actually.

Line 39: Barker (1996) is not the best reference in this case. That paper deals with horizontal inhomogeneity of single cloud layers, therefore irrelevant for GCMs. A much better reference is Oreopoulos and Barker, QJRMS (1999) which deals with multiple vertically overlapped cloudy layers each of which has a gamma distribution of LWC. That scheme was specifically designed for GCMs and was actually deployed on a couple (no papers exist though), but was quickly superseded by MclCA.

Lines 27-29: When reference is made to the maximum-random overlap assumption, everyone assumes that there is a unique implementation, but that's simply not true! Indeed, there are various flavors. Geleyn and Hollingsworth (1979) may actually be the best one. But there is actually the cloud "block" implementation of max-ran, which is visually captured in Fig. 10 of Chou et al., JAS (1998). In GH79, two cloudy layers that have another cloudy layer in-between are still assumed to be maximally overlapped for the common portion they have with the in-between layer, but the portions of the layers

Printer-friendly version

Discussion paper
Interactive

comment

C3 
that correspond to the clear fraction of the in-between layer are randomly-overlapped. In the Chou et al. representations these clouds would be maximally overlapped if they belonged to the same high, middle, or low block. Only the blocks themselves are randomly overlapped. But the Chou et al. (1998) is still called a max-ran scheme, yet is it very different than GH79! Also, incidentally, the Morcrette and Fouquart paper does not discuss or advocate for max-ran overlap. Rather, it compares, max, min, and two versions of random overlap.

Line 306, leave it to the reader. Well, it's not very common to ask such a thing! Why don't you include these other three cases in the Appendix?

And a few minor corrections:

Line 61: "pioneering"

Line 79: "exertion"? You mean version?

Line 88: "pairs".

Table 1: Odd to call the third experiment "TSM". All experiments not conducted with MC are TSM. So strictly speaking, you have TSM-ICA, TSM-HOM, and TSM-GCM. You could have also conducted the experiment in the middle field with MC, i.e, MCHOM. Would still have 3D effects because of finite cloud sizes, but no effects due to internal cloud heterogeneity.

Line 376: "validity"

Lines 378-379: No discussion of the Fig. 12 results?

Line 419: You mean rightmost column?

Line 476: Or there was no bias reduction at all!

Printer-friendly version

Lines 485-486: Another marine BL cloud classification you may want to mention is this:

Discussion paper

https://www.atmos-meas-tech-discuss.net/amt-2020-61/ 
Interactive comment on Atmos. Chem. Phys. Discuss., https://doi.org/10.5194/acp-2020-334, 2020.

Interactive comment 